\title{
Paramagnetic Centers in DOPA-Melanin-Dihydrostreptomycin Complexes
}

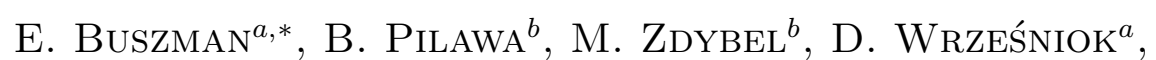
A. GrzegorczyK ${ }^{a}$ And T. WILCZOK ${ }^{c}$

${ }^{a}$ Department of Pharmaceutical Chemistry School of Pharmacy, Medical University of Silesia Jagiellońska 4, 41-200 Sosnowiec, Poland ${ }^{b}$ Department of Medical Physics School of Pharmacy, Medical University of Silesia Narcyzów 1, 41-200 Sosnowiec, Poland ${ }^{c}$ Department of Molecular Biology and Medical Genetics School of Pharmacy, Medical University of Silesia Narcyzów 1, 41-200 Sosnowiec, Poland

DOPA-melanin-dihydrostreptomycin complexes with drug concentrations $1 \times 10^{-4}-1 \times 10^{-2} \mathrm{M}$ were examined by the use of electron paramagnetic resonance spectroscopy at X-band $(9.3 \mathrm{GHz})$. Dihydrostreptomycin was chosen for studies, because this aminoglycoside antibiotic causes strong toxic effects in organism. It was stated that dihydrostreptomycin generates $o$-semiquinone free radicals with $g=2.0038$ in melanin. Free radicals formation increases with increase in the antibiotic concentration. Changes of EPR lines with microwave powers pointed out that slow spin-lattice relaxation processes exist in DOPA-melanin and in its complexes with dihydrostreptomycin. The measured EPR lines were homogeneously broadened.

PACS numbers: 61.66.Hq, 76.30.Rn

\section{Introduction}

$o$-semiquinone free radicals are responsible for stable paramagnetism of melanin biopolymers [1]. Eumelanins with characteristic single lines mainly exist in living organisms. Free radicals play an important role in biochemical reac-

*corresponding author; e-mail: ebuszman@slam.katowice.pl 
tions with drugs [2-4]. Application of aminoglycoside antibiotics therapy may be accompanied by toxic effects resulting from free radicals generation in melanin.

Dihydrostreptomycin is an aminoglycoside antibiotic which contains streptidine as aminocyclitol and two sugars: L-streptose and N-methyl-L-glucosamine. Aminocyclitol and sugars are linked by glycosidic bond.

Aminoglycosides are polycations, and their polarity is in part responsible for pharmacokinetic properties shared by all members of the group. The aminoglycoside antibiotics are used primarily to treat infections caused by aerobic gram-negative bacteria, they interfere with protein synthesis in susceptible microorganisms. Although they are widely used and important agents, serious toxicity is a major limitation to the usefulness of these antibiotics. Most notable are nephrotoxicity and ototoxicity, which can involve both the auditory and vestibular functions of the eight cranial nerve [5].

The aim of this work was to study influence of dihydrostreptomycin on free radicals in DOPA-melanin — the model eumelanin. The changes of free radical concentrations in DOPA-melanin after binding of dihydrostreptomycin were tested.

\section{Experimental}

Synthetic DOPA-melanin was formed by oxidative polymerization of 3,4-dihydroxyphenylalanine (L-DOPA) in $0.07 \mathrm{M}$ phosphate buffer at $\mathrm{pH}$ 8.0. DOPA-melanin-dihydrostreptomycin complexes [M]: $1 \times 10^{-4}, 5 \times 10^{-4}, 1 \times 10^{-3}$, $5 \times 10^{-3}$, and $1 \times 10^{-2}$, were analyzed by electron paramagnetic resonance spectroscopy.

Spectra were measured using an X-band (9.3 GHz) EPR spectrometer produced by RADIOPAN-Poznań. $g$-factors, line widths $\Delta B_{\mathrm{pp}}$, amplitudes $A$ of the spectra were determined. Free radical concentrations in the studied complexes were calculated. Ultramarine was used as the reference of paramagnetic centers concentration. Microwave saturation of EPR spectra was examined.

\section{Results and discussion}

For $o$-semiquinone free radicals $(g=2.0038)$ in DOPA-melanin and its complexes with dihydrostreptomycin broad EPR lines with widths in the range $0.45-$ $0.51 \mathrm{mT}$ were measured. Parameters of the EPR lines and concentrations of paramagnetic centers in the analyzed complexes are presented in Table I.

Free radicals concentrations in DOPA-melanin-dihydrostreptomycin complexes were higher than in DOPA-melanin (Table I). Increase in free radical concentration with increase in drug concentration in DOPA-melanin-dihydrostreptomycin complexes was observed (Tables I, II). Dipolar interactions and line widths increase for the samples with higher concentrations of complexing agents $\left(5 \times 10^{-3} \mathrm{M}, 1 \times 10^{-2} \mathrm{M}\right)$. 
TABLE I

Free radical concentration $N, g$-factor, and line widths $\Delta B_{\mathrm{pp}}$ of EPR lines of DOPA-melanin and its complexes with dihydrostreptomycin.

\begin{tabular}{c|c|c|c}
\hline \hline Drug concentration $[\mathrm{M}]$ & $N \times 10^{-19}[\mathrm{spin} / \mathrm{g}]$ & $\Delta B_{\mathrm{pp}}[ \pm 0.02 \mathrm{mT}]$ & $g[ \pm 0.0002]$ \\
\hline DOPA-melanin & 1.2 & 0.46 & 2.0039 \\
$1 \times 10^{-4}$ & 1.2 & 0.46 & 2.0038 \\
$5 \times 10^{-4}$ & 1.3 & 0.45 & 2.0038 \\
$1 \times 10^{-3}$ & 1.4 & 0.47 & 2.0038 \\
$5 \times 10^{-3}$ & 1.5 & 0.50 & 2.0038 \\
$1 \times 10^{-2}$ & 1.7 & 0.51 & 2.0039
\end{tabular}

Effect of drug concentration on the amount of dihydrostreptomycin bound to DOPA-melanin.

\begin{tabular}{c|c}
\hline \hline Drug concentration $[\mathrm{M}]$ & Amount of drug bound to melanin $[\mu \mathrm{mol} / \mathrm{mg}$ melanin $]$ \\
\hline $1 \times 10^{-4}$ & 0.0902 \\
$5 \times 10^{-4}$ & 0.3769 \\
$1 \times 10^{-3}$ & 0.5535 \\
$5 \times 10^{-3}$ & 1.1436 \\
$1 \times 10^{-2}$ & 1.1595
\end{tabular}

Influence of microwave power in the range $0.7-70 \mathrm{~mW}$ on amplitudes and line widths of EPR lines of the exemplary DOPA-melanin-dihydrostreptomycin complexes is shown in Figs. 1a and b. EPR lines of all the studied melanin com-
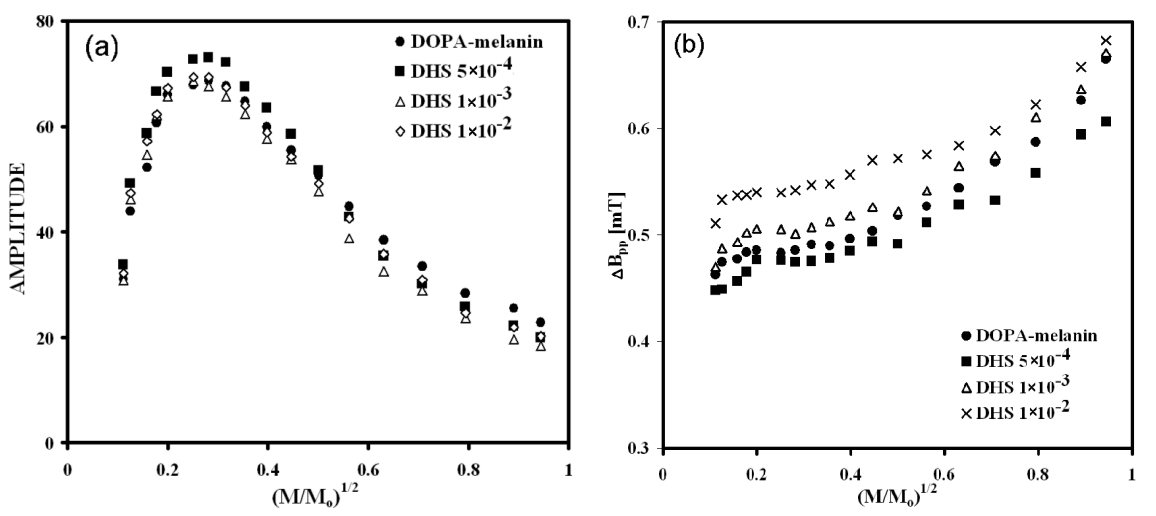

Fig. 1. Changes of amplitude $A$ (a) and line width $\Delta B_{\mathrm{pp}}$ (b) of EPR lines of DOPA-melanin and its complexes with dihydrostreptomycin (concentrations [M]: $5 \times 10^{-4}, 1 \times 10^{-3}$, and $1 \times 10^{-2}$ ) with increase in microwave power. $M-$ microwave power used during the measurement of EPR spectrum, $M_{0}$ - total microwave power $(70 \mathrm{~mW})$ produced by klystron. 
plexes saturate at low microwave powers, which indicates long spin-lattice relaxation time in melanin samples. The antibiotic concentration in DOPA-melanindihydrostreptomycin complexes does not modify the effect of microwave power on amplitude of their EPR lines. Spin-lattice relaxation times in DOPA-melanin and DOPA-melanin-dihydrostreptomycin complexes are similar. Line widths of EPR lines of the studied melanin samples increase with increase in microwave power (Fig. 1b). Changes of melanin EPR lines (amplitudes and line widths) with microwave power indicate their homogeneous broadening (Fig. 1).

\section{Conclusions}

It was obtained that free radical concentration in DOPA-melanindihydrostreptomycin complexes increases with increase in drug bound to melanin. Formation of $o$-semiquinone free radicals in DOPA-melanin-dihydrostreptomycin complexes by the aminoglycoside antibiotic may be responsible for toxic effects in organism during pharmacotherapy application.

Dihydrostreptomycin does not change spin-lattice interactions in melanin. Slow spin-lattice relaxation processes exist in the all DOPA-melanindihydrostreptomycin complexes. EPR lines of both DOPA-melanin and its complexes with dihydrostreptomycin are homogeneously broadened.

EPR lines of the studied samples saturate at low microwave power. Changes of EPR spectra of DOPA-melanin-dihydrostreptomycin complexes with microwave power indicate their homogeneous broadening.

\section{References}

[1] E. Buszman, B. Pilawa, T. Witoszyńska, M. Latocha, T. Wilczok, Appl. Magn. Res. 24, 401 (2003).

[2] B. Pilawa, E. Buszman, D. Wrześniok, M. Latocha, T. Wilczok, Appl. Magn. Res. 23, 181 (2002).

[3] B. Pilawa, M. Latocha, E. Buszman, T. Wilczok, Appl. Magn. Res. 25, 105 (2003).

[4] E. Buszman, B. Pilawa, M. Zdybel, D. Wrześniok, A. Grzegorczyk, T. Wilczok, Chem. Phys. Lett. 403, 22 (2005).

[5] J.G. Hardman, L.E. Limbird, P.B. Molinoff, R.W. Ruddon, G.A. Gilman, The Pharmacological Basis of Therapeutics, McGraw-Hill, New York 1996. 